\title{
The IncRNA BORG: a novel inducer of TNBC metastasis, chemoresistance, and disease recurrence
}

\author{
Alex J. Gooding', Kimberly A. Parker², Saba Valadkhan³, William P. Schiemann ${ }^{4}$ \\ 'Department of Pathology, Case Western Reserve University, Cleveland, OH 44106, USA. \\ 2Department of Pharmacology, Case Western Reserve University, Cleveland, OH 44106, USA. \\ ${ }^{3}$ Department of Molecular Biology and Microbiology, Case Western Reserve University, Cleveland, OH 44106, USA. \\ ${ }^{4}$ Case Comprehensive Cancer Center, Case Western Reserve University, Cleveland, OH 44106, USA.
}

Correspondence to: Dr. William P. Schiemann, Case Comprehensive Cancer Center, Case Western Reserve University, Wolstein Research Building, Room 2131, 2103 Cornell Road Cleveland, OH 44106, USA. E-mail: william.schiemann@case.edu; Dr. Saba Valadkhan, Department of Molecular Biology and Microbiology, Case Western Reserve University, Wood Building, 10900 Euclid Avenue, Cleveland, OH 44106, USA. E-mail: saba.valadkhan@case.edu

How to cite this article: Gooding AJ, Parker KA, Valadkhan S, Schiemann WP. The IncRNA BORG: a novel inducer of TNBC metastasis, chemoresistance, and disease recurrence. J Cancer Metastasis Treat 2019;5:41.

http://dx.doi.org/10.20517/2394-4722.2019.11

Received: 24 Jan 2019 First Decision: 1 Apr 2019 Revised: 12 Apr 2019 Accepted: 15 Apr 2019 Published: 10 May 2019

Science Editor: Ren Xu Copy Editor: Cai-Hong Wang Production Editor: Huan-Liang Wu

\begin{abstract}
Although greater than $90 \%$ of breast cancer-related mortality can be attributed to metastases, the molecular mechanisms underpinning the dissemination of primary breast tumor cells and their ability to establish malignant lesions in distant tissues remain incompletely understood. Genomic and transcriptomic analyses identified a class of transcripts called long noncoding RNA (IncRNA), which interact both directly and indirectly with key components of gene regulatory networks to alter cell proliferation, invasion, and metastasis. We identified a pro-metastatic IncRNA BMP/OP-Responsive Gene (BORG) whose aberrant expression promotes metastatic relapse by reactivating proliferative programs in dormant disseminated tumor cells (DTCs). BORG expression is broadly and strongly induced by environmental and chemotherapeutic stresses, a transcriptional response that facilitates the survival of DTCs. Transcriptomic reprogramming in response to BORG resulted in robust signaling via survival and viability pathways, as well as decreased activation of cell death pathways. As such, BORG expression acts as a (1) marker capable of predicting which breast cancer patients are predisposed to develop secondary metastatic lesions; and (2) unique therapeutic target to maximize chemosensitivity of DTCs. Here we review the molecular and cellular factors that contribute to the pathophysiological activities of BORG during its regulation of breast cancer metastasis, chemoresistance, and disease recurrence.
\end{abstract}

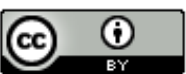

(C) The Author(s) 2019. Open Access This article is licensed under a Creative Commons Attribution 4.0 International License (https://creativecommons.org/licenses/by/4.0/), which permits unrestricted use, sharing, adaptation, distribution and reproduction in any medium or format, for any purpose, even commercially, as long as you give appropriate credit to the original author(s) and the source, provide a link to the Creative Commons license, and indicate if changes were made.

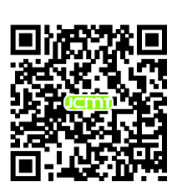


Keywords: BORG, chemoresistance, disease recurrence, dormancy, long noncoding RNA, metastasis, triple-negative breast cancer

\section{INTRODUCTION}

With over 250,000 newly diagnosed cases in 2017, breast cancer is the most commonly diagnosed malignancy among U.S. women ${ }^{[1]}$. Although recent advances in treatment have produced a moderate decline in the mortality rate associated with breast cancer $^{[2]}$, this disease nevertheless still plagues women and is the second leading cause of cancer-related death, resulting in over 40,000 deaths each year ${ }^{[1]}$. The clinical management of breast cancer is complicated by its manifestation as an exceptionally heterogeneous disease. In fact, the degree of molecular and histopathologic variation demonstrated by breast cancers necessitates its classification not as a single, uniform disease, but rather as one that is composed of a diverse collection of diseases that possess varying clinical prognoses and require distinct treatment strategies. Integral to this heterogeneity are the receptors for the ovarian steroid hormones estrogen (ER) and progesterone (PR), and for the membrane-associated tyrosine kinase HER2/ErbB-2. Moreover, the expression or lack thereof of these receptors dictates therapeutic schemes and disease-free progression ${ }^{[3,4]}$. Likewise, the multiple various permutations in the expression patterns of these receptors exemplify the heterogeneity of the disease and necessitate the tailoring of specific treatments to each individual patient. In fact, clinicopathologic detection for the expression of these receptors remains an advantageous trait, as they represent some of the most consistently predictive and actionable molecular targets in all of oncology ${ }^{[5]}$. As such, the largest clinical burden associated with breast cancer stems from a subset of patients whose tumors fail to express ER, PR, and HER2, lesions known as triple-negative breast cancer (TNBC). This genetically distinct breast cancer subtype constitutes $\sim 15 \%-20 \%$ of all diagnosed breast cancers ${ }^{[6]}$ and portends the worst overall survival rates of all breast cancer subtypes, an untoward trait that reflects their extreme propensity to relapse within 5 years of initial diagnosis and treatment ${ }^{[7]}$. The molecular features that underlie the development, metastasis, and relapse of TNBCs remain to be fully elucidated. Recently, a novel intergenic lncRNA known as BMP/ OP-Responsive Gene (BORG) was identified as being a prominent driver of these tumorigenic activities in TNBCs. Here we highlight the pathophysiology associated with aberrant BORG expression in TNBCs, as well as discuss clinical implications of BORG and its potential for therapeutic targeting to alleviate metastatic disease.

\section{Breast cancer metastasis}

Despite immense efforts undertaken to characterize the molecular complexity of primary breast cancers, the lethality associated with all subtypes of breast cancer is attributed primarily to the dissemination and colonization of distant tissues ${ }^{[8,9]}$, an untoward clinical event that results in dismal median survival rates of $\sim 1$ year. Moreover, the finding of metastatic TNBC is essentially a fatal diagnosis regardless of the chemotherapeutic intervention deployed to combat this disease ${ }^{[10]}$. Despite decades of intense investigation, a complete understanding of the molecular forces vital to metastasis remains incomplete, a knowledge gap that continues to hinder the development of therapeutics capable of specifically targeting and alleviating metastatic lesions. Nonetheless, fundamental steps taken by malignant cells to facilitate their dissemination from the primary tumor site to distant tissues for colonization have been identified and are called the metastatic cascade ${ }^{[11]}$. The events that comprise this multi-step process include: (1) local invasion and migration of malignant cells into the stroma surrounding the primary lesion; (2) intravasation into the vasculature or lymphatic system to permit transit through these circulatory routes; (3) vascular stasis and subsequent extravasation into parenchymal tissues at distant sites of colonization; (4) survival and micrometastasis formation; and (5) overt growth and metastasis formation ${ }^{[12]}$. Interestingly, the earliest phases of metastatic progression (i.e., dissociation and emigration from site of origin via the vasculature) represent the barriers that are most easily surmounted by primary lesions, as evidenced by the fact that tumors readily liberate thousands upon thousands of cells into the circulation each day ${ }^{[13,14]}$. However, 
despite the relative abundance of tumor cells traversing the vascular systems of some patients, the process of metastasis remains a supreme inefficient event. Indeed, animal modeling studies estimate that only $\sim 0.01 \%$ of circulating tumor cells are capable of initiating some form of metastatic outgrowth ${ }^{[15]}$, and as such, it is the late stages of metastatic progression that ultimately dictate the competency of disseminated cells in establishing overt metastases within the metastatic niche ${ }^{[12]}$.

\section{Dormancy: a metastatic bottleneck}

The acquisition of metastatic phenotypes was originally believed to be an evolutionary consequence of advanced, late-stage disease. However, recent findings indicate that carcinoma cells comprising primary breast tumors do in fact enter the circulation months-to-years prior to the point at which the primary tumor becomes symptomatic and diagnosable ${ }^{[16]}$. As such, a large proportion of breast cancer patients already harbor disseminated tumor cells (DTCs) at their time of diagnosis ${ }^{[11,17,18]}$. Indeed, DTCs are readily detected in the bone marrow of $30 \%-40 \%$ of patients diagnosed with early-stage breast cancer, an event that portends a significantly worse prognosis as compared to patients whose bone marrow is free from DTCs at the time of diagnosis ${ }^{[19]}$. Interestingly, a large fraction of these DTCs initially remain clinically asymptomatic due in part to their acquisition of dormancy-associated phenotypes upon arrival to the micrometastatic niche ${ }^{[12]}$.

Although diverse genetic and epigenetic analyses have begun to reveal the molecular landscape that characterizes metastatic breast cancers, only recently have these investigations been directed at and tailored for DTCs and their reactivation of proliferative programs during metastatic relapse. As such, a unified definition of dormancy remains elusive. At a cellular level, current models suggest that metastatic cells often undergo proliferative arrest upon arriving to a micrometastatic niche, a phenomenon believed to reflect the initial maladaptation of DTCs to foreign stromal environments ${ }^{[20]}$. Indeed, the stromal characteristics that impact DTC dormancy are multifactorial and encompass a diverse array of immunomodulatory and vascular endothelial cell signals (e.g., cytokine milieu, rigidity of the microenvironment, presence of active immunosurveillance ${ }^{[17,21,22]}$ ) that converge on niche-localized DTCs. In doing so, these unique microenvironment signals may prove to be inhospitable to DTCs, thereby provoking cellular stress and the initiation of apoptosis as these cells struggle to cope and respond to foreign junctional and adhesive signaling networks ${ }^{[23-25]}$. Consequently, newly established micrometastatic lesions fail to propagate and expand due to: (1) deficiencies to initiate and progress through the cell cycle; and (2) propensity to undergo apoptosis at a pace that equals or exceeds the proliferative rate of DTCs. Despite these barriers, a subset of DTCs remain viable and poised to reactivate proliferative programs that result in metastatic relapse yearsto-decades after implementation of initial treatments, such as chemotherapy and radical primary tumor resection ${ }^{[26,27]}$. Clinically, extended periods of metastatic dormancy is evidenced by growth modeling studies performed on over 1,000 breast cancer patients. In doing so, two discrete peaks that define the probability of metastatic recurrence were identified: (1) one correlating with a model of continuous, slow growth of metastatic cells; and (2) one corroborating the principle that the majority of delayed relapses are indeed the result of a temporary period of dormancy prior to reactivation of proliferation programs ${ }^{[28]}$. Indeed, it is this second peak that poses the greatest threat to breast cancer patients, with $\sim 62 \%$ of breast cancer deaths occurring 5-20 years after initial diagnosis ${ }^{[27]}$. Taken together, these findings reveal that dormant DTCs play a pivotal role in the majority of breast cancer-associated mortality, a feature that cements them as one of the most clinically relevant targets in all of oncology.

\section{MODELS OF METASTATIC DORMANCY IN BREAST CANCER}

In vitro and in vivo models of dormancy represent critical tools for investigating the molecular mediators that impact dormant states. However, the establishment of such models pose significant challenges, as the growth and propagation of dormant cell lines is, by definition, inherently impractical. Likewise, the size and sparsity of dormant micrometastases makes their identification highly burdensome. Nonetheless, accepted models of breast cancer dormancy do in fact exist. One particularly powerful model of metastatic dormancy 
is the murine D2.HAN series, which consists of two cell lines that display distinct metastatic properties, namely the dormant D2.OR cell line and the highly metastatic D2.A1 cell line ${ }^{[29]}$. These cell lines were derived from a premalignant murine hyperplastic alveolar nodule implanted into the cleared mammary fat pad of $B A L B / c$ mice, resulting in spontaneous tumors that were subsequently classified by alternations in their surface glycoprotein composition as determined by retention to the lectin, peanut agglutinin ${ }^{[2]}$.

Accordingly, dormant D2.OR cells exhibit high affinity for peanut agglutinin and produce slow growing tumors that are incapable of forming disseminated tumors in either spontaneous or experimental metastasis models. In stark contrast, metastatic D2.A1 cells exhibit low affinity for peanut agglutinin and produce fast growing tumors that metastasize aggressively ${ }^{[30]}$. Importantly, in vivo videomicroscopy reveals that these D2.HAN derivatives show no differences in the mechanism, timing, and proportion of cells capable of extravasating into the lungs of mice, indicating that post-extravasation events underlie their vastly disparate abilities to undergo metastatic outgrowth ${ }^{[31]}$. Despite their inability to form secondary tumors, a large proportion of disseminated D2.OR cells readily survive the process of extravasation (i.e., $\sim 80 \%$ at 3 weeks and $\sim 50 \%$ at 11 weeks) and remain viable despite their non-proliferative phenotype (i.e., Ki-67-negative) ${ }^{[32]}$. Collectively, these cellular and functional features form the crux of the dormancy-associated phenotypes exhibited by D2.OR cells in vivo, thus establishing them as a valuable model for studying the molecular mechanisms underlying metastatic dormancy ${ }^{[33-35]}$.

\section{IncRNAs in breast cancer}

Elucidating the molecular determinants of breast cancer metastasis, especially those associated with DTC acquisition of and eventual emergence from dormancy, remains a critical undertaking that is essential to the future development of therapies capable of targeting DTCs. Recently, a surprising class of molecules called long noncoding RNAs (lncRNAs) have been identified and function as potent contributors to the malignant properties of breast cancer cells. Broadly, these transcripts are defined as RNA molecules that are $>200$ nucleotides in length and lack an open reading frame capable of producing a functional protein ${ }^{[36]}$. LncRNAs were originally believed to possess negligible function and exist primarily as "transcriptional noise" originating from illegitimate regulatory DNA elements. However, it is now clear that lncRNA expression patterns transpire in a highly specific cell- and tissue-dependent manner ${ }^{[37,38]}$. Importantly, noncoding RNA molecules do not require protein-coding capacity to act as powerful determinants of cell fate. Indeed, lncRNAs harbor immense intrinsic functionality within the course of cellular homeostasis and disease formation. For instance, the developmental complexity of organisms correlates more closely with the extent and diversity of the noncoding genome rather than with the collective composition of protein-coding genes ${ }^{[39-41]}$.

In light of the dynamic and diverse functions attributed to lncRNAs, it is unsurprising that malignant cells, including those arising from the breast, have hijacked lncRNAs to directly and indirectly alter their proliferative, invasive, and metastatic ability ${ }^{[42-44]}$. As such, several lncRNAs have been shown to modify critical breast cancer-associated molecular pathways in a manner that transcends hormone receptor status, frequently driving the development and progression of $\mathrm{TNBCs}^{[45]}$. Similarly, ER-associated signaling pathways also regulate the expression of $\operatorname{lncRNAs}{ }^{[46]}$, including HOTAIR, whose promoter contains several estrogen response elements ${ }^{[4]}$.

Moreover, induction of HOTAIR promotes the growth and metastasis of breast cancers via widespread epigenetic reprogramming ${ }^{[48]}$.

\section{BORG}

Although numerous IncRNAs have been linked to breast cancer tumorigenesis and metastasis ${ }^{[49]}$, the intergenic lncRNA BORG has recently emerged as a unique and formidable regulator of the metastatic competence and survival of breast cancer cells. Originally discovered in murine $\mathrm{C}_{2} \mathrm{C} 12$ myoblast cells treated with $\mathrm{BMP} 2$ or $\mathrm{BMP} 7, \mathrm{BORG}$ is a spliced and polyadenylated $\sim 2.8 \mathrm{~kb}$ transcript that shows no 
evidence of an open reading frame and carries multiple conserved repeat sequence elements of unclear significance ${ }^{[50]}$. Collectively, these features implicate BORG as a lncRNA, whose primary sequence has been subject to several functional analyses. For instance, BORG houses several novel pentamer motifs that are essential in facilitating its strict residence in the nucleus, representing the first demonstration of sequencebased determinants operant in dictating the subcellular localization of lncRNAs ${ }^{[51]}$. Indeed, the nuclear localization of lncRNAs directly impacts their ability to elicit widespread alterations in transcriptional networks by: (1) localizing transcription factors to specific genomic loci ${ }^{[52-54]}$; and (2) exerting gross changes in the nuclear architecture of cells ${ }^{[55]}$. Likewise, BORG oversees a host of cellular functions that are readily harnessed by breast cancer cells to enhance their tumorigenic behaviors. As will be discussed in the succeeding sections, these BORG-dependent events play an essential role in promoting breast cancer cell proliferation, chemoresistance, and survival.

\section{Control of proliferation}

In undertaking a combination of in silico and cell biological analyses, we recently determined that the expression of BORG directly correlates with aggressive breast cancer phenotypes, and with their metastatic competence and recurrence. Specifically, BORG liberates D2.OR cells from a state of dormancy in $3 \mathrm{D}$-organotypic cultures by conferring a proliferative shift in the cell cycle from $\mathrm{Go} / \mathrm{G} 1$ to $\mathrm{G} 2 / \mathrm{S}^{[56]}$. Importantly, this proliferative stimulus is sufficient to enable BORG-expressing D2.OR cells to form overt metastases in the lungs of BALB/c mice ${ }^{[56]}$. Interestingly, the mitogenic properties of BORG are highly context-dependent, as they only emerge in D2.OR cells propagated in microenvironments that mimic primary and/or metastatic tumor sites (e.g., 3D-cultures). Such context-dependent activities of BORG imply that this lncRNA confers malleable phenotypes to DTCs, thus compelling them to activate adaptive signaling programs that enable their survival and outgrowth within diverse metastatic niches.

\section{BORG as a manipulator of protein function}

In searching for mechanistic insights into how BORG induces DTCs to escape from dormancy, we performed mass spectrometry analyses on proteins captured by the pulldown of biotinylated, anti-sense BORG transcripts. These analyses identified the E3 SUMO ligase TRIM28 (KAP1) as a strong binding partner of BORG ${ }^{[57]}$. TRIM28 functions as a transcriptional co-repressor and scaffolding protein for histone and DNA modifying enzymes that enhance breast cancer cell proliferation, doing so in part by suppressing the transcription of senescence promoting genes, especially $p 21$ and Gadd45 $a^{[58-61]}$. Interestingly, elevating BORG expression in D2.OR cells to levels that approximate those detected in their metastatic D2.A1 counterparts dramatically downregulated the expression $p 21$ and Gadd45a, indicating that BORG may rely upon TRIM28 to confer proliferative states to dormant DTCs. Indeed, CRISPR/Cas9-mediated knockout of TRIM28 restores a dormant phenotype in BORG-expressing D2.OR cells, as does expression of mutant BORG transcripts that can no longer bind TRIM2 ${ }^{[57]}$. Thus, the oncogenic activities of BORG depend upon its physical interaction with TRIM28, an event that serves as a proliferative stimulus to dormant DTCs.

The diverse range of functions elicited by lncRNAs is thought to be promoted by their unique structural diversity. Their inherent length and nucleic acid structure allow the formation of flexible, complex secondary and higher order structures that facilitate their interactions with macromolecular complexes ${ }^{[40]}$. Indeed, lncRNAs can acquire behaviors analogous to ligands, as their binding to proteins can trigger conformational changes and/or modify protein: protein interactions that dramatically impact protein activation states ${ }^{[62]}$. Accordingly, BORG enhances the function of TRIM28 to inhibit transcription by regulating the pause and release of RNA Polymerase II (Pol II $)^{[63,64]}$. For instance, heterologous expression of mutant BORG transcripts that retain their capacity to bind TRIM28 remain competent to elicit Pol II promoter pausing at the 21 and Gadd45a loci, whereas those BORG mutants incapable of binding TRIM28 fail to impact the pausing index of Pol II at these loci ${ }^{[57]}$. Furthermore, widespread evidence indicates that lncRNAs can function as molecular scaffolds for proteins, thereby: (1) tethering cooperative proteins together to enhance their functions; or (2) localizing RNA-protein complexes to specific genomic regions through base-pair and 
tertiary structure interactions with $\mathrm{DNA}^{[41,65]}$. Along these lines, chromatin immunoprecipitation assays reveal that BORG enhances the binding of TRIM28 to specific genomic loci ${ }^{[57]}$. Thus, BORG promotes the metastatic outgrowth of dormant DTCs in part through its ability to promote the localization and transcriptional repressive activity of TRIM28. Although BORG directly modifies the ability of TRIM28 to suppress the expression of $p 21$ and gadd45a in dormant DTCs, it should be noted that TRIM28 also exerts widespread alterations in the transcriptomes of a multitude of cell types ${ }^{[63,64,66]}$. To gain additional insight into the repertoire of transcriptional events coupled to TRIM28 in DTCs, we performed RNA-seq and microarray-based transcriptomic analyses on parental and BORG-expressing D2.OR organoids propagated in $3 \mathrm{D}$-cultures. To assess the specific impact of TRIM28 on these dormancy-associated phenotypes (i.e., parental dormancy $v s$. BORG-mediated outgrowth), we also rendered these cells deficient in TRIM28 expression. Unsurprisingly, BORG-expressing D2.OR cells harbored a transcriptional signature that deviated significantly from its parental and TRIM28-deficient counterparts. Moreover, cellular network analyses revealed specific BORG- and TRIM28-dependent transcriptional patterns that were significantly enriched for proliferative and pro-metastatic signatures ${ }^{[57]}$. Collectively, these findings establish BORG as the only known lncRNA that functions in modifying the activity and cellular localization of a transcriptional regulator (i.e., TRIM28) to confer genome-wide transcriptomic alterations that compel the reactivation of proliferative programs in dormant DTCs.

\section{Chemoresistance}

The development of therapeutic resistances continues to hamper the prolonged efficacy of standard-of-care treatment regimens. Moreover, these clinical challenges are compounded by the fact that the underlying mechanisms responsible for targeted and chemotherapeutic resistance are immensely diverse. Nonetheless, malignant breast cancer cells regularly rely on the malleable intrinsic state of cancer cells, which enables their adaptation to cytotoxic cellular stresses in order to maintain viability ${ }^{[67]}$ in a manner that most closely follows the paradigm of acquired resistance ${ }^{[68]}$. Indeed, the plasticity underlying the appearance of chemoresistance is naturally permissive and reflects alterations in the epigenome. Moreover, these events are bolstered by defects in the ability of DTCs to maintain genome integrity that arise in response to aberrant cell cycle checkpoints and DNA repair mechanisms, and to increased rates of proliferation. Additionally, interactions between DTCs and the tumor microenvironment induce unique de novo mechanisms of therapeutic resistance, as cell adhesion networks (e.g., integrins) activate a specialized survival program known as "cell adhesion-mediated" drug resistance that elicit DTC insensitivity to numerous treatment regimens ${ }^{[69,70]}$. As such, the stromal composition of the metastatic microenvironment creates a natural sanctuary for DTCs to survive therapeutic insults.

Interestingly, dormant DTCs have long been recognized for their inherent resistance to commonly used chemotherapeutic drugs ${ }^{[11]}$. These resistant traits naturally stem from the quiescent phenotype of dormant cells, which effectively abolishes the clinical utility of chemotherapeutics and cytotoxic agents that target metabolically active and dividing cells ${ }^{[71]}$. Moreover, dormant cells preferentially upregulate signaling pathways associated with cell survival, a trait stemming from their allocation of metabolic resources away from cell cycle progression as a means to remain viable in the face of environmental stressors (i.e., chemotherapy $)^{[72]}$. Accordingly, chemotherapeutic treatment can select for a subset of dormant cells that are enriched for pro-survival pathways and multidrug resistance, implying that cytotoxic insults can select for a population of cells that are exceedingly equipped to instigate post-therapy relapse ${ }^{[73,74]}$. Importantly, we recently determined that BORG plays a central role in driving the development of chemoresistance in TNBCs (see below).

\section{BORG: a novel inducer of chemoresistance}

In addition to possessing enhanced proliferative abilities, BORG-expressing D2.OR cells also exhibit: (1) extensive upregulation of pro-survival and viability pathways; and (2) widespread downregulation of cell 
Table 1. BORG-associated signaling pathways

\begin{tabular}{lclll}
\hline Upstream inducers & BORG-complexes & Downstream effectors & \multicolumn{1}{c}{ Cellular response } & Ref. \\
\hline BMP; OP-1; TGF- $\beta$ & BORG & Alkaline phospatase & Osteoblast Differentiation & {$[50]$} \\
NF-kB & BORG & NF-kB & Cellular Stress Response/Cell survival & {$[56,84,85]$} \\
ATM*:\# & BORG:TRIM28 & P21* & Proliferation & {$[57,58]$} \\
ATM*.\# $^{*}$ & BORG:TRIM28 & Gadd45a* $^{*}$ & Proliferation & {$[57,58]$} \\
- & BORG:TRIM28 & P38/MAPK* & Proliferation & {$[57]$} \\
- & BORG:TRIM28 & ERK1/2 & Proliferation & {$[57]$} \\
Hypoxia/chemostresses & BORG:RPA1 & RPA2 \& RPA3 & DNA Damage Response & {$[56,80]$} \\
Hypoxia/chemostresses & BORG:RPA1 & BRCA2 $^{*}$ & DNA Damage Response & {$[56,81-83]$} \\
Hypoxia/chemostresses & BORG:RPA1 & XPA $^{*}$ & DNA Damage Response & {$[56,81-83]$} \\
Hypoxia/chemostresses & BORG:RPA1 & P53 $^{\#}$ & DNA Damage Response & {$[56,81-83]$} \\
\hline
\end{tabular}

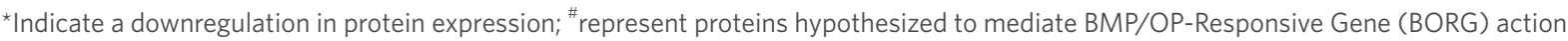
based on findings published in the literature. BORG plays essential functions in regulating breast cancer proliferation, metastasis, and chemoresistance. The specific effectors of BORG that activate cells proliferation and survival systems are annotated above, as are the BORG:effector complexes operant in mediating these events

death pathways ${ }^{[56]}$. Moreover, BORG expression is highly responsive to metabolic stresses such as hypoxia and nutrient deprivation, as well as to treatment with a wide panel of chemotherapeutic drugs, including doxorubicin, hydroxyurea, docetaxel, 5-fluorouracil, and 6-thioguanine ${ }^{[56]}$. These cellular attributes of BORG are consistent with its ability to enhance metastatic outgrowth and disease recurrence. Indeed, comet assays demonstrate that heterologous expression of BORG dramatically mitigates the extent of doublestranded DNA breaks experienced by D2.OR cells in response to doxorubicin exposure. Moreover, these genoprotective features of BORG rely upon its binding to the single-strand DNA-binding protein, RPA1, which functions as an essential molecule in the repair of DNA damage ${ }^{[75,76]}$. Interestingly, the capacity of BORG to induce resistance to doxorubicin appears to supersede its ability to enhance cell cycle progression, an event that could potentially render BORG-expressing cells more sensitive to the cytotoxic activities of doxorubicin ${ }^{[77,78]}$. As such, the genoprotective effects of BORG are essential in establishing the foundation operant in mediating deadly relapse in patients with metastatic breast cancers. Moreover, as lncRNAs typically function as molecular scaffolds that facilitate the formation of protein complexes, particularly in the nucleus ${ }^{[79]}$, it is tempting to speculate that BORG promotes the interaction of RPA1 with proteins critical to its repair of DNA, including additional subunits RPA2 and RPA $3^{[80]}$, as well as other DNA-repair associated proteins, such as BRCA2, XPA, and p53 ${ }^{[81-83]}$ [Table 1].

\section{BORG and NF-KB: a feed-forward loop to chemoresistance}

Cellular network analyses of parental and BORG-expressing D2.OR derivatives demonstrate significant enrichment of a hallmark gene signature that correlates with the induction of NF- $\mathrm{KB}$ activity ${ }^{[5]}$. Accordingly, $\mathrm{NF}-\kappa \mathrm{B}$ reporter assays show enhanced activation of NF- $\kappa \mathrm{B}$ signaling in BORG-expressing D2.OR cells as compared to their parental counterparts. In light of the longstanding association between NF- $\kappa \mathrm{B}$ activation and the initiation of pro-survival and cellular stress responses ${ }^{[84,85]}$, we further explored the connections between NF- $\mathrm{KB}$ and BORG in eliciting DTC resistance to chemotherapy. In doing so, we inactivated the NF$\kappa \mathrm{B}$ pathway via several complementary strategies, including: (1) stable expression of a dominant-negative form of IKB $\alpha$; (2) CRISPR/Cas9-mediated knockout of the BORG-responsive gene NEMO/IKK $\gamma$; or (3) administration of small molecule inhibitors to IKK $\beta$. In all cases, inhibiting NF- $\mathrm{KB}$ signaling in BORGexpressing D2.OR cells restored their sensitivity to doxorubicin both in vitro and in vivo, and to apoptosis induced by hypoxia- and nutrient deprivation ${ }^{[56]}$. It should be noted that while BORG requires ample expression of RPA1 to protect against doxorubicin-induced DNA damage and subsequent apoptosis, this event appears to be dissociated from the ability of BORG to activate NF- $\mathrm{B}$ signaling. Indeed, CRISPR/Cas9mediated disruption of RPA1 expression had no effect on the ability of BORG to activate NF- $\mathrm{KB}$ signaling ${ }^{[56]}$. Thus, the chemoresistant phenotypes afforded by BORG-RPA1 interactions act independently of the NF- $\mathrm{B}$ signaling axis. 
Interestingly, the promoter region of the BORG locus contains multiple stretches of nucleotides that exhibit sequence homology to the consensus DNA-binding sequence for NF- $\mathrm{B}$. Accordingly, NF- $\mathrm{B}$ B readily bound to the BORG promoter in BORG-expressing cells, thereby identifying a novel feed-forward loop whereby the activation of NF- $\mathrm{B}$ (i.e., through chemotherapeutic and environmental stressors ${ }^{[86-89]}$ ) leads to enhanced BORG expression, which further promotes the induction NF- $\kappa \mathrm{B}$ responsive genes. Along these lines, expression of a dominant-negative I $\mathrm{KB} \alpha$ in TNBCs prevented their expression of BORG following exposure to doxorubicin, and to environmental stresses, such as hypoxia and nutrient deprivation ${ }^{[56]}$. Collectively, these findings implicate BORG as a unique lncRNA that is capable of promoting a NF- $\kappa \mathrm{B}$ feed-forward signaling loop that effectively links metastasis-associated cellular stresses to a coordinated signaling program that engenders the survival of disseminated TNBCs.

\section{BORG and breast cancer stem cells}

Breast cancer stem cells (BCSCs) are malignant cells capable of tumor initiation, self-renewal, and differentiation into a heterogeneous group of cancer cells that reflect those present in the original primary breast tumor; they are also associated with the acquisition of metastatic and chemoresistant phenotypes ${ }^{[90-93]}$, particularly upon their colonization of foreign tissue microenvironments ${ }^{[94]}$. BCSCs typically divide asymmetrically to create: (1) a single progenitor cell capable of differentiating into a variety of functionally diverse cancer cell types; and (2) a single BCSC that can expand and undergo continual self-renew. Attempts to characterize BCSCs has been hampered by a relative lack of universal markers for BCSCs ${ }^{[95,96]}$. Indeed, BCSCs have been linked to the expression of several cell surface proteins, such as CD133 and CD $44^{+} / \mathrm{CD} 24$ , and to the intracellular protein, ALDH $1^{[97-99]}$. Recent findings have associated the expression of lncRNAs with the generation and expansion of BCSCs. For instance, the lncRNAs ROR, HOTAIR, and Hh all induce the expression of transcription factors that regulate "stemness", such as SOX2 and OCT4; they also impact the initiation of EMT programs ${ }^{[100-103]}$, which elicit the selection, expansion, and self-renewal of BCSCs ${ }^{[104]}$. The role of BCSCs in regulating metastasis, chemoresistance, and survival signaling are reminiscent of the features attributed to aberrant BORG expression, suggesting that BORG may also regulate the behaviors of BSCSs. Accordingly, BORG expression is elevated significantly in BCSCs as compared to non-BCSCs populations; it also enhances the mammosphere forming activity of human and murine breast cancer cells (Gooding et al ${ }^{[56,57]}$, unpublished observation). Thus, future studies need to determine precisely how BORG impacts the transcriptomic and epigenetic landscapes of breast cancer cells to impart BCSC characteristics coupled to metastatic progression and disease recurrence.

\section{DISCUSSION AND CLINICAL IMPLICATIONS OF BORG}

Metastasis is an exceedingly complicated process, whereby tumor cells must undergo coordinated efforts to successfully disperse from primary tumors, emigrate to distant tissues, and survive and colonize foreign microenvironments. Even for highly transformed cells, traversing the metastatic cascade is immensely challenging, with the vast majority of disseminated cells unable to form overt metastases ${ }^{[15]}$. The inefficiencies characterizing metastasis are predominantly attributed to the cellular stresses associated with unfamiliar metastatic microenvironments, forces that obstruct both the survival and outgrowth of DTCs. Accordingly, BORG has emerged as a potent and unique lncRNA that is poised to enhance breast cancer metastasis by altering both sides of this equation, i.e., providing both proliferative and pro-survival stimuli to DTCs.

From a potential clinical perspective, aberrant BORG expression is most frequently associated with TNBC/ basal-like breast cancers, as determined by scrutinizing several publicly available RNA-seq datasets. Moreover, we detected significant elevations of BORG in metastatic human patient-derived xenograft tissue samples, and in CNS metastases and their matched primary tumors from which these metastatic foci derived. In all cases, malignant tissues clearly express increased levels of BORG as compared to normal human mammary epithelial cells ${ }^{[5,57]}$. Thus, these findings support the hypothesis that primary 
malignancies that house high levels of BORG will disperse aggressive breast cancer cells that are predisposed to establishing clinically-relevant, chemoresistant secondary lesions. Indeed, TNBC patients who succumbed to metastatic relapse within 5 years of initial diagnosis and treatment possessed primary tumors that contained significantly higher levels of BORG compared to primary tumors derived from TNBC patients who remained disease-free for at least 5 years post-treatment ${ }^{[57]}$. Taken together, these intriguing findings suggest that quantifying BORG expression in primary tumors could offer important insights into predicting the natural and clinical course of breast disease within TNBC patients.

The correlative finding that BORG expression largely aligns with the overall malignant propensity of breast cancer cells fails to address the mechanisms and signaling systems ultimately coupled to its upregulation in developing mammary tumors. Indeed, it has been proposed that lncRNAs evolved as a means to assist in maintaining cellular homeostasis in response to a wide variety of pathophysiologic conditions ${ }^{[105]}$. Accordingly, we show that BORG expression is similarly influenced by a number of cellular stressors, including chemotherapeutic insult, nutrient deprivation, and hypoxia ${ }^{[56]}$, as well as in response to heat shock (Gooding et al. ${ }^{[56]}$ and Valadkhan et al. ${ }^{[105]}$, unpublished observation). Moreover, NF- $\kappa \mathrm{B}$ is critical in linking the responsiveness of BORG to these environmental stressors ${ }^{[56]}$, as NF- $\mathrm{KB}$ activation has long been tied to the survival of cells confronted with a host of intrinsic and extrinsic stressors ${ }^{[84,85,106]}$. It therefore stands to reason that increased flux through the $\mathrm{NF}_{-} \mathrm{KB}$ pathway stemming from exposure to noxious stimuli may

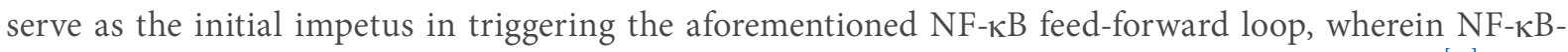
induced upregulation of BORG propagates expression of NF- $\kappa \mathrm{B}-$ responsive gene expression patterns ${ }^{[56]}$.

Tumor progression and metastatic competence are thought to rely heavily upon the intrinsic plasticity of malignant cells, which facilitates their adaptation to harsh foreign microenvironments in order to maintain viability ${ }^{[67,107]}$. Because BORG is largely regulated by extracellular paracrine factors (e.g., TGF- $\beta$, BMP-2, and BMP- ${ }^{[50,56]}$ ) and the environmental cues, we surmise that BORG acts as a context-dependent, transcriptional rheostat for disseminated breast cancer cells, thereby dictating their engagement of proliferative and prosurvival pathways. Indeed, when faced with environmental or therapeutic stresses, the induction of BORG in malignant cells orchestrates a transcriptional signature that provokes aggressive tumorigenic states that ensures for their survival. As such, preventing BORG expression or impeding the activation of its downstream targets represents an innovative and potentially impactful strategy to target metastatic breast cancers and drive them into an apoptosis-prone state.

Current mechanistic insights into the regulation of BORG and its downstream effectors reveal that this lncRNA is uniquely poised to promote the metastasis of breast cancer cells. Indeed, BORG clearly exerts a pro-metastatic effect at both the primary and metastatic sites of tumor growth. For example, a subset of breast cancer patients may harbor primary tumor cells that have gained the expression of BORG (BORG $\left.{ }^{\text {hi }}\right)$ as a result of environmental stresses associated with a growing primary tumor (i.e., hypoxia and nutrient deprivation; Figure 1). Such tumors are prone to shedding BORG ${ }^{\text {hi }}$ cells into the circulation that disseminate to distant tissues, wherein they exploit the proliferative and pro-survival effects of BORG to overcome the hostile metastatic microenvironment and form overt metastases [Figure 1]. Accordingly, and as noted above, TNBC patients who succumbed to metastatic relapse within 5 years of initial diagnosis and treatment possessed primary tumors that contained significantly higher levels of BORG compared to primary tumors derived from TNBC patients who remained disease-free for at least 5 years post-treatment. Furthermore, breast cancer cells can emigrate from the primary tumor at very early stages of tumor development ${ }^{[18]}$. As such, these early disseminated breast cancer cells are likely to originate from a lesion experiencing little hypoxic or metabolic stress and are therefore more prone to harboring low levels of BORG (BORG ${ }^{\text {lo }}$. Although still capable of disseminating to distant tissues, these $\mathrm{BORG}^{\text {lo }}$ cells are predicted to struggle within their foreign microenvironments, resulting in their undergoing cell death or retreating into a state of metastatic dormancy. Nonetheless, stromal paracrine signals (e.g., TGF- $\beta$, BMP2, or BMP7), as well as the 


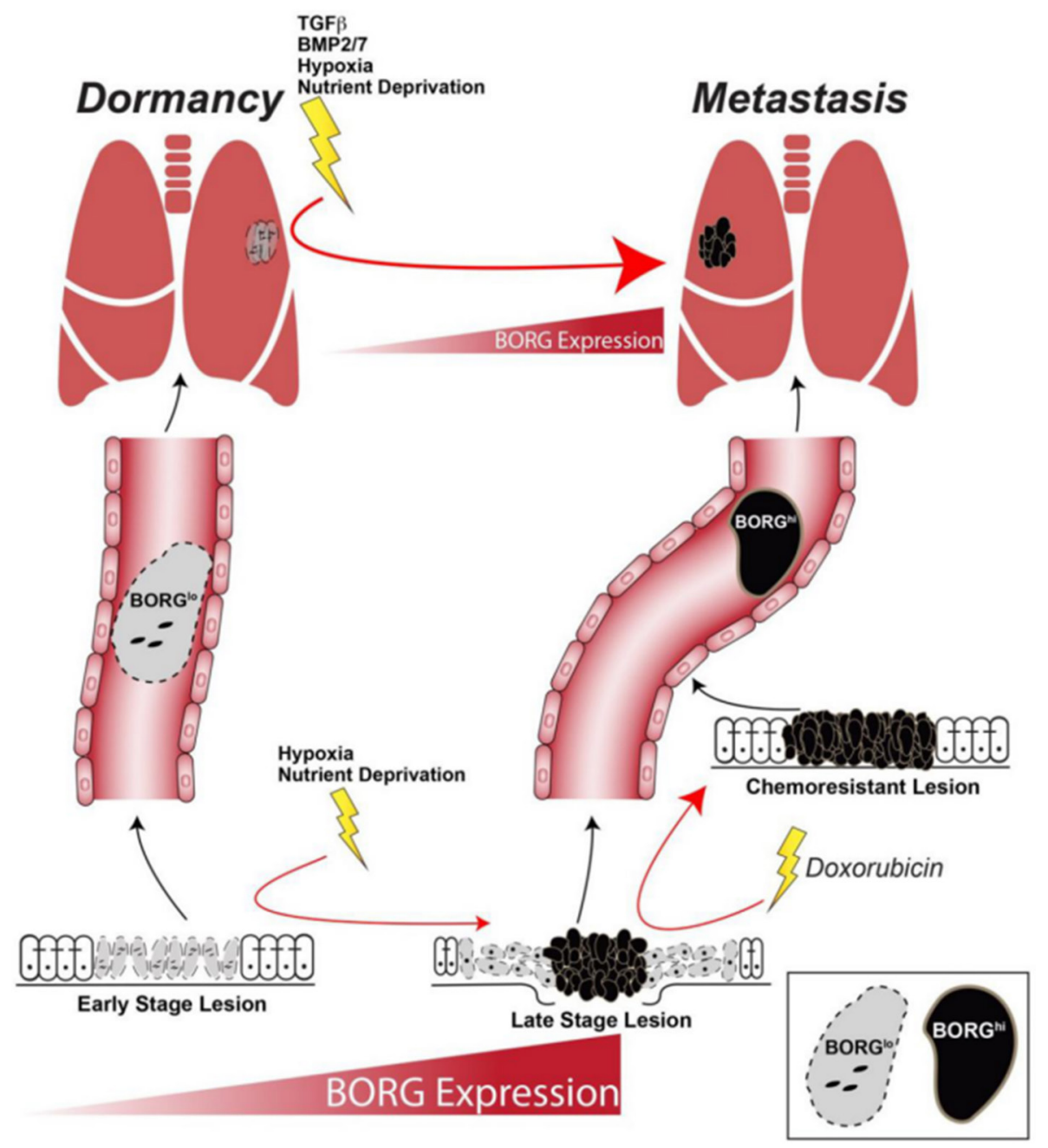

Figure 1. BORG is a potent facilitator of breast cancer metastasis. Breast cancer cells can disseminate at very early stages of development. Due to the benign microenvironment associated with these lesions, these cells are likely to harbor low levels of BORG $\left(B O R G^{\circ}\right)$ expression and are prone to establishing dormant lesions in metastatic tissues. Nonetheless, stromal factors and environmental stressors in the metastatic microenvironment can induce BORG expression, thereby compelling these dormant cells to reinstate proliferative programs. The progression of primary tumors is associated with a hypoxic environment and stark competition for nutrients. Such stresses can enhance BORG expression in a subset of cells (BORG ${ }^{\text {hi }}$. These BORG hi cells can disseminate to distant tissues where they exploit the proliferative and survival advantages afforded by BORG to produce overt metastases. Cytotoxic chemotherapeutic treatment is effective against $B O R G^{10}$ cells, but BORG ${ }^{\text {hi }}$ cells show resistance to such therapies and are the foundation for chemoresistant, residual disease that can eventually metastasize to distant tissues. BORG: BMP/OP-Responsive Gene

hypoxic and metabolic stresses associated with the metastatic microenvironment, can lead to the induction of BORG expression within these dormant DTCs, thereby activating proliferative programs and survival signaling to promote their metastatic outgrowth [Figure 1].

Finally, neoadjuvant or adjuvant treatment of primary breast cancers with chemotherapeutic agents (e.g., doxorubicin) will selectively kill BORG ${ }^{\text {lo }}$ cells that are not inherently resistant to the cytotoxic effects of these agents. The residual, chemoresistant BORG ${ }^{\text {hi }}$ cells can subsequently contribute to the recurrence of metastatic or primary lesions that are insensitive to standard-of-care therapies [Figure 1]. These diverse cellular outcomes downstream of BORG establish this lncRNA as an essential driver of breast cancer metastasis and highlight the potential utility derived from therapeutically targeting BORG or its effectors as a novel means to alleviate the metastatic outgrowth and recurrence of disseminated TNBCs. 


\section{DECLARATIONS}

\section{Acknowledgments}

Members of the Schiemann Laboratory are thanked for critical comments and reading of the manuscript.

\section{Authors' contributions}

Conception and study design: Gooding AJ, Parker KA, Schiemann WP

Drafted and revised the manuscript: Gooding AJ, Parker KA, Valadkhan S, Schiemann WP

\section{Availability of data and materials}

Not applicable.

\section{Financial support and sponsorship}

Research support was provided in part by the National Institutes of Health (CA236273) to Schiemann WP and Valadkhan S, and (T32GM007250 and F30CA203233) to Gooding AJ. Additional support was graciously provided by the METAvivor Foundation to Schiemann WP, and by pilot funding from the Case Comprehensive Cancer Center's Research Innovation Fund, which is supported by the Case Council and Friends of the Case Comprehensive Cancer Center to Schiemann WP.

\section{Conflicts of interest}

All authors declare that there are no conflicts of interest.

\section{Ethical approval and consent to participate}

Not applicable.

\section{Consent for publication}

Not applicable.

\section{Copyright}

(c) The Author(s) 2019.

\section{REFERENCES}

1. Siegel RL, Miller KD, Jemal A. Cancer statistics, 2018. CA Cancer J Clin 2019;69:7-34.

2. Welch HG, Prorok PC, O'Malley AJ, Kramer BS. Breast-cancer tumor size, overdiagnosis, and mammography screening effectiveness. N Engl J Med 2016;375:1438-47.

3. Arendt LM, Kuperwasser C. Form and function: how estrogen and progesterone regulate the mammary epithelial hierarchy. J Mammary Gland Biol Neoplasia 2015;20:9-25.

4. Gingras I, Gebhart G, de Azambuja E, Piccart-Gebhart M. HER2-positive breast cancer is lost in translation: time for patient-centered research. Nat Rev Clin Oncol 2017;14:669-81.

5. Payne SJ, Bowen RL, Jones JL, Wells CA. Predictive markers in breast cancer--the present. Histopathology 2008;52:82-90.

6. Anders CK, Carey LA. Biology, metastatic patterns, and treatment of patients with triple- negative breast cancer. Clin Breast Cancer 2009;9:S73-81.

7. Costa RLB, Gradishar WJ. Triple-negative breast cancer: current practice and future directions. J Oncol Pract 2017;13:301-3.

8. Nguyen DX, Massague J. Genetic determinants of cancer metastasis. Nat Rev Genet 2007;8:341-52.

9. Gupta GP, Massague J. Cancer metastasis: building a framework. Cell 2006;127:679-95.

10. Dent R, Trudeau M, Pritchard KI, Hanna WM, Kahn HK, et al. Triple-negative breast cancer: clinical features and patterns of recurrence. Clin Cancer Res 2007; 13:4429-34.

11. Pantel K, Brakenhoff RH. Dissecting the metastatic cascade. Nat Rev Cancer 2004;4:448- 56.

12. Vanharanta S, Massague J. Origins of metastatic traits. Cancer Cell 2013;24:410-21.

13. Stott SL, Lee RJ, Nagrath S, Yu M, Miyamoto DT, et al. Isolation and characterization of circulating tumor cells from patients with localized and metastatic prostate cancer. Sci Transl Med 2010;2:25ra23.

14. Nagrath S, Sequist LV, Maheswaran S, Bell DW, Irimia D, et al. Isolation of rare circulating tumour cells in cancer patients by microchip technology. Nature 2007;450:1235-9.

15. Luzzi KJ, MacDonald IC, Schmidt EE, Kerkvliet N, Morris VL, et al. Multistep nature of metastatic inefficiency: dormancy of solitary 
cells after successful extravasation and limited survival of early micrometastases. Am J Pathol 1998;153:865-73.

16. Hosseini H, Obradovic MM, Hoffmann M, Harper KL, Sosa MS, et al. Early dissemination seeds metastasis in breast cancer. Nature 2016;540:552-8.

17. Sosa MS, Bragado P, Aguirre-Ghiso JA. Mechanisms of disseminated cancer cell dormancy: an awakening field. Nat Rev Cancer 2014;14:611-22.

18. van 't Veer LJ, Dai H, van de Vijver MJ, He YD, Hart AA, et al. Gene expression profiling predicts clinical outcome of breast cancer. Nature 2002;415:530-6.

19. Hartkopf AD, Taran FA, Wallwiener M, Hahn M, Becker S, et al. Prognostic relevance of disseminated tumour cells from the bone marrow of early stage breast cancer patients - results from a large single-centre analysis. Eur J Cancer 2014;50:2550-9.

20. Aguirre-Ghiso JA. Models, mechanisms and clinical evidence for cancer dormancy. Nat Rev Cancer 2007;7:834-46.

21. MacKie RM, Reid R, Junor B. Fatal melanoma transferred in a donated kidney 16 years after melanoma surgery. N Engl J Med 2003;348:567-8.

22. Ghajar CM, Peinado H, Mori H, Matei IR, Evason KJ, et al. The perivascular niche regulates breast tumour dormancy. Nat Cell Biol 2013;15:807-17.

23. Holmgren L, O'Reilly MS, Folkman J. Dormancy of micrometastases: balanced proliferation and apoptosis in the presence of angiogenesis suppression. Nature medicine 1995;1:149- 53.

24. Shibue T, Weinberg RA. Integrin beta1-focal adhesion kinase signaling directs the proliferation of metastatic cancer cells disseminated in the lungs. Proc Natl Acad Sci U S A 2009;106:10290-5.

25. Shibue T, Brooks MW, Inan MF, Reinhardt F, Weinberg RA. The outgrowth of micrometastases is enabled by the formation of filopodiumlike protrusions. Cancer Discov 2012;2:706-21.

26. Karrison TG, Ferguson DJ, Meier P. Dormancy of mammary carcinoma after mastectomy. J Natl Cancer Inst 2000;92:1101A-101.

27. Klein CA. Framework models of tumor dormancy from patient-derived observations. Curr Opin Genet Dev 2011;21:42-9.

28. Demicheli R, Retsky MW, Hrushesky WJ, Baum M. Tumor dormancy and surgery-driven interruption of dormancy in breast cancer: learning from failures. Nat Clin Pract Oncol 2007;4:699-710.

29. Rak JW, McEachern D, Miller FR. Sequential alteration of peanut agglutinin binding- glycoprotein expression during progression of murine mammary neoplasia. Br J Cancer 1992;65:641-8.

30. Morris VL, Tuck AB, Wilson SM, Percy D, Chambers AF. Tumor progression and metastasis in murine D2 hyperplastic alveolar nodule mammary tumor cell lines. Clin Exp Metastasis 1993;11:103-12.

31. Morris VL, Koop S, MacDonald IC, Schmidt EE, Grattan M, et al. Mammary carcinoma cell lines of high and low metastatic potential differ not in extravasation but in subsequent migration and growth. Clin Exp Metastasis 1994;12:357-67.

32. Naumov GN, MacDonald IC, Weinmeister PM, Kerkvliet N, Nadkarni KV, et al. Persistence of solitary mammary carcinoma cells in a secondary site: a possible contributor to dormancy. Cancer Res 2002;62:2162-8.

33. De Cock JM, Shibue T, Dongre A, Keckesova Z, Reinhardt F, et al. Inflammation triggers zeb-dependent escape from tumor latency. Cancer Res 2016;76:6778-84

34. Vera-Ramirez L, Vodnala SK, Nini R, Hunter KW, Green JE. Autophagy promotes the survival of dormant breast cancer cells and metastatic tumour recurrence. Nat Commun 2018;9:1944.

35. Albrengues J, Shields MA, Ng D, Park CG, Ambrico A, et al. Neutrophil extracellular traps produced during inflammation awaken dormant cancer cells in mice. Science 2018;361:pii:eaao4227.

36. Rinn JL, Chang HY. Genome regulation by long noncoding RNAs. Annu Rev Biochem 2012;81:145-66.

37. Djebali S, Davis CA, Merkel A, Dobin A, Lassmann T, et al. Landscape of transcription in human cells. Nature 2012;489:101-8.

38. Ponting CP, Oliver PL, Reik W. Evolution and functions of long noncoding RNAs. Cell 2009;136:629-41.

39. Mattick JS, Taft RJ, Faulkner GJ. A global view of genomic information--moving beyond the gene and the master regulator. Trends Genet 2010;26:21-8

40. Fatica A, Bozzoni I. Long non-coding RNAs: new players in cell differentiation and development. Nat Rev Genet 2014;15:7-21.

41. Guttman M, Rinn JL. Modular regulatory principles of large non-coding RNAs. Nature 2012;482:339-46.

42. Huarte M. The emerging role of IncRNAs in cancer. Nat Med 2015;21:1253-61.

43. Cabanski CR, White NM, Dang HX, Silva-Fisher JM, Rauck CE, et al. Pan-cancer transcriptome analysis reveals long noncoding RNAs with conserved function. RNA Biol 2015;12:628-42.

44. Iyer MK, Niknafs YS, Malik R, Singhal U, Sahu A, et al. The landscape of long noncoding RNAs in the human transcriptome. Nat Genet 2015;47:199-208.

45. Jiang YZ, Liu YR, Xu XE, Jin X, Hu X, et al. Transcriptome analysis of triple-negative breast cancer reveals an integrated mRNAlncRNA signature with predictive and prognostic value. Cancer Res 2016;76:2105-14.

46. Hah N, Danko CG, Core L, Waterfall JJ, Siepel A, et al. A rapid, extensive, and transient transcriptional response to estrogen signaling in breast cancer cells. Cell 2011;145:622-34.

47. Bhan A, Hussain I, Ansari KI, Kasiri S, Bashyal A, et al. Antisense transcript long noncoding RNA (lncRNA) HOTAIR is transcriptionally induced by estradiol. J Mol Biol 2013;425:3707-22.

48. Gupta RA, Shah N, Wang KC, Kim J, Horlings HM, et al. Long non-coding RNA HOTAIR reprograms chromatin state to promote cancer metastasis. Nature 2010;464:1071-6.

49. Richard JLC, Eichhorn PJA. Deciphering the roles of lncRNAs in breast development and disease. Oncotarget 2018;9:20179-212.

50. Takeda K, Ichijo H, Fujii M, Mochida Y, Saitoh M, et al. Identification of a novel bone morphogenetic protein-responsive gene that may function as a noncoding RNA. J Biol Chem 1998;273:17079-85.

51. Zhang B, Gunawardane L, Niazi F, Jahanbani FK, Chen X, et al. A novel RNA motif mediates the strict nuclear localization of a long noncoding RNA. Mol Cell Biol 2014;34:2318-29. 
52. Derrien T, Johnson R, Bussotti G, Tanzer A, Djebali S, et al. The GENCODE v7 catalog of human long noncoding RNAs: analysis of their gene structure, evolution, and expression. Genome Res 2012;22:1775-89.

53. Geisler S, Coller J. RNA in unexpected places: long non-coding RNA functions in diverse cellular contexts. Nat Rev Mol Cell Biol 2013;14:699-712.

54. Mercer TR, Mattick JS. Structure and function of long noncoding RNAs in epigenetic regulation. Nat Struct Mol Biol 2013;20:300-7.

55. Kopp F, Mendell JT. Functional classification and experimental dissection of long noncoding RNAs. Cell 2018;172:393-407.

56. Gooding AJ, Zhang B, Gunawardane L, Beard A, Valadkhan S, et al. The IncRNA BORG facilitates the survival and chemoresistance of triple-negative breast cancers. Oncogene 2019;38:2020-41.

57. Gooding AJ, Zhang B, Jahanbani FK, Gilmore HL, Chang JC, et al. The lncRNA BORG drives breast cancer metastasis and disease recurrence. Sci Rep 2017;7:12698.

58. Li X, Lee YK, Jeng JC, Yen Y, Schultz DC, et al. Role for KAP1 serine 824 phosphorylation and sumoylation/desumoylation switch in regulating KAP1-mediated transcriptional repression. The Journal of biological chemistry 2007;282:36177-89.

59. Lee YK, Thomas SN, Yang AJ, Ann DK. Doxorubicin down-regulates Kruppel-associated box domain-associated protein 1 sumoylation that relieves its transcription repression on p21WAF1/CIP1 in breast cancer MCF-7 cells. J Biol Chem 2007;282:1595-606.

60. Addison J, Koontz C, Fugett JH, Creighton CJ, Chen D, et al. KAP1 promotes proliferation and metastatic progression of breast cancer cells. Cancer Res 2015;75:344-55.

61. Calderon MR, Verway M, Benslama RO, Birlea M, Bouttier M, et al. Ligand-dependent corepressor contributes to transcriptional repression by $\mathrm{C} 2 \mathrm{H} 2$ zinc-finger transcription factor ZBRK1 through association with KRAB-associated protein-1. Nucleic Acids Res 2014;42:7012-27.

62. Deb M, Kar S, Sengupta D, Shilpi A, Parbin S, et al. Chromatin dynamics: H3K4 methylation and H3 variant replacement during development and in cancer. Cell Mol Life Sci 2014;71:3439-63.

63. Bunch H, Zheng X, Burkholder A, Dillon ST, Motola S, et al. TRIM28 regulates RNA polymerase II promoter-proximal pausing and pause release. Nat Struct Mol Biol 2014;21:876-83.

64. Hu G, Kim J, Xu Q, Leng Y, Orkin SH, et al. A genome-wide RNAi screen identifies a new transcriptional module required for selfrenewal. Genes Dev 2009;23:837-48.

65. Batista PJ, Chang HY. Long noncoding RNAs: cellular address codes in development and disease. Cell 2013;152:1298-307.

66. Iyengar S, Farnham PJ. KAP1 protein: an enigmatic master regulator of the genome. J Biol Chem 2011;286:26267-76.

67. Crea F, Danesi R, Farrar WL. Cancer stem cell epigenetics and chemoresistance. Epigenomics 2009;1:63-79.

68. Quintieri L, Fantin M, Vizler C. Identification of molecular determinants of tumor sensitivity and resistance to anticancer drugs. Adv Exp Med Biol 2007;593:95-104.

69. Dalton WS. The tumor microenvironment as a determinant of drug response and resistance. Drug Resist Updat 1999;2:285-8.

70. Hazlehurst LA, Landowski TH, Dalton WS. Role of the tumor microenvironment in mediating de novo resistance to drugs and physiological mediators of cell death. Oncogene 2003;22:7396-402.

71. Naumov GN, Townson JL, MacDonald IC, Wilson SM, Bramwell VH, et al. Ineffectiveness of doxorubicin treatment on solitary dormant mammary carcinoma cells or late-developing metastases. Breast Cancer Res Treat 2003;82:199-206.

72. Ranganathan AC, Adam AP, Zhang L, Aguirre-Ghiso JA. Tumor cell dormancy induced by p38SAPK and ER-stress signaling: an adaptive advantage for metastatic cells? Cancer Biol Ther 2006;5:729-35.

73. Dai Y, Wang L, Tang J, Cao P, Luo Z, et al. Activation of anaphase-promoting complex by p53 induces a state of dormancy in cancer cells against chemotherapeutic stress. Oncotarget 2016;7:25478-92.

74. Kleffel S, Schatton T. Tumor dormancy and cancer stem cells: two sides of the same coin? Adv Exp Med Biol 2013;734:145-79.

75. Zhao M, Geng R, Guo X, Yuan R, Zhou X, et al. PCAF/GCN5-mediated acetylation of RPA1 promotes nucleotide excision repair. Cell Rep 2017;20:1997-2009.

76. Haring SJ, Mason AC, Binz SK, Wold MS. Cellular functions of human RPA1. Multiple roles of domains in replication, repair, and checkpoints. J Biol Chem 2008;283:19095-111.

77. Baral E, Auer G. In vitro effect of doxorubicin on non-proliferating and proliferating epithelial cells. Int J Radiat Oncol Biol Phys 1990;19:963-5.

78. Li S, Kennedy M, Payne S, Kennedy K, Seewaldt VL, et al. Model of tumor dormancy/recurrence after short-term chemotherapy. PLoS One 2014;9:e98021.

79. Ribeiro DM, Zanzoni A, Cipriano A, Delli Ponti R, Spinelli L, et al. Protein complex scaffolding predicted as a prevalent function of long non-coding RNAs. Nucleic Acids Res 2018;46:917-28.

80. Bochkareva E, Korolev S, Lees-Miller SP, Bochkarev A. Structure of the RPA trimerization core and its role in the multistep DNA-binding mechanism of RPA. EMBO J 2002;21:1855-63.

81. Wong JM, Ionescu D, Ingles CJ. Interaction between BRCA2 and replication protein A is compromised by a cancer-predisposing mutation in BRCA2. Oncogene 2003;22:28-33.

82. Romanova LY, Willers H, Blagosklonny MV, Powell SN. The interaction of $\mathrm{p} 53$ with replication protein A mediates suppression of homologous recombination. Oncogene 2004;23:9025-33.

83. Li L, Lu X, Peterson CA, Legerski RJ. An interaction between the DNA repair factor XPA and replication protein A appears essential for nucleotide excision repair. Mol Cell Biol 1995;15:5396-402.

84. Wang T, Zhang X, Li JJ. The role of NF-kappaB in the regulation of cell stress responses. Int Immunopharmacol 2002;2:1509-20.

85. Mercurio F, Manning AM. NF-kappaB as a primary regulator of the stress response. Oncogene 1999;18:6163-71.

86. Hoesel B, Schmid JA. The complexity of NF-kappaB signaling in inflammation and cancer. Mol Cancer 2013;12:86.

87. Hayden MS, Ghosh S. NF-kappaB, the first quarter-century: remarkable progress and outstanding questions. Genes Dev 2012;26:203-34.

88. Godwin P, Baird AM, Heavey S, Barr MP, O'Byrne KJ, et al. Targeting nuclear factor-kappa B to overcome resistance to chemotherapy. 
Front Oncol 2013;3:120.

89. Montagut C, Tusquets I, Ferrer B, Corominas JM, Bellosillo B, et al. Activation of nuclear factor-kappa B is linked to resistance to neoadjuvant chemotherapy in breast cancer patients. Endocr Relat Cancer 2006;13:607-16.

90. Liu H, Patel MR, Prescher JA, Patsialou A, Qian D, et al. Cancer stem cells from human breast tumors are involved in spontaneous metastases in orthotopic mouse models. Proc Natl Acad Sci U S A 2010;107:18115-20.

91. Pece S, Tosoni D, Confalonieri S, Mazzarol G, Vecchi M, et al. Biological and molecular heterogeneity of breast cancers correlates with their cancer stem cell content. Cell 2010;140:62-73.

92. Louie E, Nik S, Chen JS, Schmidt M, Song B, et al. Identification of a stem-like cell population by exposing metastatic breast cancer cell lines to repetitive cycles of hypoxia and reoxygenation. Breast Cancer Res 2010;12:R94.

93. Dongre A, Weinberg RA. New insights into the mechanisms of epithelial-mesenchymal transition and implications for cancer. Nat Rev Mol Cell Biol 2019;20:69-84.

94. Li F, Tiede B, Massague J, Kang Y. Beyond tumorigenesis: cancer stem cells in metastasis. Cell Res 2007;17:3-14.

95. Prager BC, Xie Q, Bao S, Rich JN. Cancer stem cells: the architects of the tumor ecosystem. Cell Stem Cell 2019;24:41-53.

96. Schatton T, Frank NY, Frank MH. Identification and targeting of cancer stem cells. Bioessays 2009;31:1038-49.

97. Jaksch M, Munera J, Bajpai R, Terskikh A, Oshima RG. Cell cycle-dependent variation of a CD133 epitope in human embryonic stem cell, colon cancer, and melanoma cell lines. Cancer Res 2008;68:7882-6.

98. Velasco-Velazquez MA, Popov VM, Lisanti MP, Pestell RG. The role of breast cancer stem cells in metastasis and therapeutic implications. Am J Pathol 2011;179:2-11.

99. Sin WC, Lim CL. Breast cancer stem cells-from origins to targeted therapy. Stem Cell Investig 2017;4:96.

100. Zhou M, Hou Y, Yang G, Zhang H, Tu G, et al. LncRNA-Hh strengthen cancer stem cells generation in twist-positive breast cancer via activation of hedgehog signaling pathway. Stem Cells 2016;34:55-66.

101. Padua Alves C, Fonseca AS, Muys BR, de Barros ELBR, Burger MC, et al. Brief report: The lincRNA Hotair is required for epithelial-tomesenchymal transition and stemness maintenance of cancer cell lines. Stem Cells 2013;31:2827-32.

102. Chen YM, Liu Y, Wei HY, Lv KZ, Fu P. Linc-ROR induces epithelial-mesenchymal transition and contributes to drug resistance and invasion of breast cancer cells. Tumour Biol 2016;37:10861-70.

103. Chen S, Zhu J, Wang F, Guan Z, Ge Y, et al. LncRNAs and their role in cancer stem cells. Oncotarget 2017;8:110685-92.

104. Mani SA, Guo W, Liao MJ, Eaton EN, Ayyanan A, et al. The epithelial-mesenchymal transition generates cells with properties of stem cells. Cell 2008;133:704-15.

105. Valadkhan S, Valencia-Hipolito A. IncRNAs in stress response. Curr Top Microbiol Immunol 2016;394:203-36.

106. Piva R, Belardo G, Santoro MG. NF-kappaB: a stress-regulated switch for cell survival. Antioxid Redox Signal 2006;8:478-86.

107. Kroger C, Afeyan A, Mraz J, Eaton EN, Reinhardt F, et al. Acquisition of a hybrid E/M state is essential for tumorigenicity of basal breast cancer cells. Proc Natl Acad Sci U S A 2019;116:7353-62. 